\title{
Health-enhancing physical activity among Saudi adults using the International Physical Activity Questionnaire (IPAQ)
}

\author{
Hazzaa M Al-Hazzaa* \\ Exercise Physiology Laboratory, King Saud University, PO Box 9792, Riyadh 11423 , Saudi Arabia
}

Submitted 7 September 2005: Accepted 31 January 2006

\begin{abstract}
Objectives: To describe the physical activity profile of Saudi adults living in Riyadh, using the International Physical Activity Questionnaire (IPAQ) short-version telephone format.

Methods: Physical activity was assessed using the official Arabic short form of IPAQ, intended for use in telephone interview. The instrument asks for times spent in walking, moderate- and vigorous-intensity physical activity of at least $10 \mathrm{~min}$ duration. The sample consisted of 1616 Saudis, between 15 and 78 years of age, living in Riyadh. Participants were drawn from a list of names in the telephone book using a simple random method. Telephone interviews were administered during the spring of 2003 by trained male interviewers.

Results: The final sample size was 1064 Saudi males and females (response rate of $66 \%$ ), with males comprising about $66 \%$ of the respondents. Over $43 \%$ of Saudis did not participate in any type of moderate-intensity physical activity lasting for at least $10 \mathrm{~min}$. More than $72 \%$ of the sample did not engage in any type of vigorous-intensity physical activity lasting for at least $10 \mathrm{~min}$. The proportion of Saudis who walked for 150 min or more per week was 33.3\%. Females were engaged more in moderate physical activity than males, whereas males participated more in vigorous activity compared with females. Activity levels did not show significant relationships with education level or job hours per week. Based on the three activity categories established by IPAQ, $40.6 \%$ of Saudis were inactive, $34.3 \%$ were minimally active and $25.1 \%$ were physically active. Physical inactivity increased with advancing age.

Conclusion: The data suggest that the prevalence of physical inactivity among Saudis adults is relatively high. Efforts are needed to encourage Saudis to be more physically active, with the goal of increasing the proportion of Saudis engaging in healthenhancing physical activity.
\end{abstract}

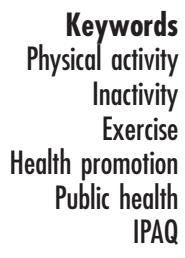

Non-communicable diseases are rapidly emerging to replace communicable diseases in developing countries ${ }^{1}$. It is believed that reducing the burden of non-communicable diseases depends on controlling several modifiable risk factors, including physical inactivity ${ }^{2}$. Indeed, physical inactivity is considered a major risk factor for a number of chronic diseases, including cardiovascular diseases, diabetes mellitus, obesity, osteoporosis and certain types of cancer $^{2-5}$. Lack of physical activity is also linked to premature mortality ${ }^{6}$. Quantitative estimates at global level indicate that sedentary living causes about $22 \%$ of ischaemic heart disease cases and about $10-16 \%$ of cases each of diabetes mellitus and breast, colon and rectal cancers ${ }^{2}$. Physical activity, on the other hand, is associated with numerous health benefits, including cardiovascular risk reduction ${ }^{3,7,8}$. Consequently, monitoring physical activity levels in society is increasingly becoming a public health priority ${ }^{9,10}$.

Previous physical activity assessment conducted in Saudi Arabia indicated that physical inactivity is becoming more prevalent among the Saudi population ${ }^{11-14}$. Such high prevalence of inactivity in Saudi Arabia represents a major public health burden, as evident by the high population-attributable risk of physical inactivity compared with many industrial countries ${ }^{15}$. Early physical activity reports in Saudi Arabia had primarily focused on leisure-time physical activity with little emphasis on other components of physical activity, i.e. occupational, transport and household/yard physical activity ${ }^{13}$. Moreover, the physical activity pattern of females was not assessed in previous local surveys ${ }^{12-14}$.

Recently, the International Physical Activity Questionnaire (IPAQ) was developed by a group of researchers from various countries, with support of the World Health Organization (WHO) and the US Centers for Disease Control and Prevention (CDC) ${ }^{16}$. The unique feature of IPAQ is that it assesses all believed health-related physical activities that can take place in different settings. There are two versions of IPAQ (a long form and a short form). In each version, 
there are two formats: self-administered and telephone or face-to-face interview. It was developed as an instrument for cross-national monitoring of physical activity and inactivity. IPAQ was subjected to a reliability and validity study carried out in 14 centres in 12 countries during the year 2000, which demonstrated that IPAQ instruments have acceptable measurement properties for monitoring population levels of physical activity among 18- to 65-year-old adults in diverse settings ${ }^{16}$. Therefore, the purpose of the present paper is to report the physical activity profile of Saudi adults living in Riyadh, using the short version of IPAQ intended for use in telephone interview.

\section{Materials and methods}

\section{Study sample}

The sample in the present study comprised 1616 Saudis aged between 15 and 78 years living in Riyadh. Riyadh is the capital of Saudi Arabia and includes people from all regions of the country. The subjects were drawn from names listed in the telephone book using a simple random method. The selected participants were contacted and interviewed via telephone by trained male interviewers during March and April of 2003. The interviewers were trained in how to introduce themselves and the subject to the participants and how to properly execute the telephone interview. Out of 1616 people who were originally selected and contacted, we were able to interview 1064 persons (about 66\% response rate). Those who were not interviewed either refused to participate in the study $(12.5 \%$ of males and $24.0 \%$ of females) or could not be reached after three attempts to reach them on three separate occasions (no answer or not found at home).

\section{Physical activity assessment}

Physical activity was assessed using the official Arabic short-version telephone format of IPAQ, which is available at www.ipaq.ki.se. The short form of IPAQ that was used in the present study has seven items providing information on time spent walking, in vigorous- and moderateintensity physical activities and in sedentary activity during the previous seven days. IPAQ defines moderate physical activities as those that produce a moderate increase in respiration rate, heart rate and sweating for at least $10 \mathrm{~min}$ duration. This is equivalent to 3-6 metabolic equivalents (MET) based on the compendium of physical activity ${ }^{17}$. Vigorous physical activities are defined as those producing vigorous increases in respiration rate, heart rate and sweating for at least $10 \mathrm{~min}$ duration. The metabolic equivalent value is above $6 \mathrm{MET}^{17}$. In addition to physical activity questions, IPAQ includes some other questions on age, education years, employment and job hours. Participants are asked to refer to all domains of physical activity including occupational, transport, household, yard/garden and leisure/sports. The questionnaires were translated and adapted to Arabic language, and then subjected to back-translation.

\section{Data analysis}

After inspecting the data, they were entered into a personal computer and checked again for outliers, according to the guidelines from IPAQ (www.ipaq.ki.se). Data were then analysed using the SPSS program, version 10.0 (SPSS Inc., Chicago, IL, USA). Frequencies and percentages were computed for each demographic characteristic, as well as for all levels of walking, moderate and vigorous physical activities. Chi-square statistics were calculated for both males and females by activity status. Levels of physical activity were examined across age groups. The relationships between activity levels and each of age group, years of education and job hours per week were tested using Spearman's Rho correlation. In additions, MET-min per week for each of walking, moderate- and vigorousintensity activities were calculated as follows: walking $=(3.3 \times$ walking minutes $\times$ walking days $)$; moderate activity $=(4.0 \times$ moderate activity minutes $\times$ moderate activity days $)$; vigorous activity $=(8.0 \times$ vigorous activity minutes $\times$ vigorous activity days). Furthermore, sufficient vigorous activity was computed on the basis of 3 or more days of vigorous-intensity activity of at least 20 min per day. Likewise, sufficiently moderate and walking activities were computed based on 5 or more days of moderate-intensity and walking of at least 30 min per day. Physical activity levels were also classified into three categories: inactive, minimally active and health-enhancing physically active, according to the scoring system provided by IPAQ (www.ipaq.ki.se).

\section{Results}

Demographic characteristics of the sample are shown in Table 1 . Almost $66 \%$ of the participants were males. Twothirds of the non-respondents were female. There was no significant difference between the mean age of males $(38.7 \pm 14.9$ years $)$ and females $(36.7 \pm 11.1$ years $)$. In general, males were more educated than females. About two-thirds of males and one-fifth of females were working. The average working hours per week were much higher for males than for females $(36.8 \pm 11.0$ vs. $\left.29.1 \pm 7.1 \mathrm{hweek}^{-1}\right)$. Among the whole group, there was a significant negative relationship $(r=-0.26$; $P<0.01)$ between age and education level. Table 2 shows the proportions of the sample that were engaging in walking, moderate- and vigorous-intensity physical activities, based on the number of days per week. Roughly, $20 \%$ of males and $27 \%$ of females in Riyadh were not walking for at least $10 \mathrm{~min}$ at a time on any single day of the week. However, over 31\% of males and 25\% of females were walking regularly every day. Moreover, about half of the males and more than a quarter of the females were not participating at all in any type of 
Table 1 Demographic characteristics of the participants $(n=1064)$

\begin{tabular}{lcc}
\hline Variable & Males & Females \\
\hline Number of participants & 702 & 362 \\
Age distribution (\%) & & \\
15-29 years & 32.7 & 29.3 \\
30-44 years & 29.5 & 44.0 \\
45-59 years & 26.5 & 23.8 \\
60-78 years & 11.3 & 2.9 \\
Education level (\%) & & \\
0 years & 3.7 & 15.4 \\
or more years & 87.5 & 68.3 \\
12 or more years & 45.0 & 30.0 \\
16 or more years & 9.6 & 4.0 \\
Employment (\%) & & \\
Employed & 63.0 & 19.3 \\
Job hours per week (\%) & & \\
20 or less & 11.6 & 35.7 \\
21-40 & 63.3 & 61.4 \\
41-60 & 17.6 & 2.9 \\
$\quad 60$ & 7.5 & 0.0 \\
\hline
\end{tabular}

moderate-intensity physical activity lasting for at least 10 min. A greater proportion of females (39.8\%) than of males (5.6\%) were doing moderate physical activity on a daily basis. With regard to vigorous activity, there were more females $(87.7 \%)$ than males $(63.8 \%)$ who were not engaging at all in vigorous-intensity physical activity lasting for at least $10 \mathrm{~min}$.

The distribution of walking, moderate and vigorous physical activities based on the number of minutes per week is shown in Table 3. Males in general spent more time in walking activity than females. The percentage of males (37.8\%) who walk for 150 min or more per week was much higher than for females (28.5\%). Further, there were more males (51.9\%) than females (27.5\%) not engaging at all in any amount of moderate physical activity, whereas more females (88.3\%) than males (64.4\%) were not taking part at all in any amount of vigorous-intensity physical activity. The proportion of females $(57.1 \%)$ who were participating in moderate-intensity physical activity for $150 \mathrm{~min}$ or more per week was much higher than of males (15.2\%). On the other hand, there were more males (13.1\%) than females (2.5\%) engaging in vigorous physical activity for $150 \mathrm{~min}$ or more per week.

The findings of the present study showed that the proportion of Saudi adults from Riyadh who were sufficiently vigorously active, based on 3 or more days of vigorous-intensity activity of at least 20 min per day, was 11.3\% (15.6\% for males and 2.8\% for females). Furthermore, the percentage of the Saudis in this study who reported sufficient moderate and walking activities, based on 5 or more days of moderate-intensity activity and walking of at least 30 min per day, was 52.8\% (47.1\% for males and $64.1 \%$ for females). Table 4 summarises the physical activity profile of the Saudi males and females, based on three activity categories. More than $40 \%$ of the Saudis were inactive, whereas about 34\% and 25\% were minimally active and active, respectively. Inactivity was slightly higher in males. Females were engaging more than males in health-enhancing physical activity.

Figure 1 shows the proportions of Saudi adults from Riyadh who were participating in health-enhancing physical activity according to age group. Physical activity increased slightly from $28.4 \%$ at age $15-29$ years to reach $29.1 \%$ at age 30-44 years. After that, there was steady drop in activity levels with advancing age to reach a low of $11.2 \%$ at age $60-78$ years. Activity category was positively related to age group ( $r=0.14 ; P<0.01$ ). There was a significant negative correlation between age and the proportion of sufficiently vigorous activity $(r=-0.22 ; P<0.01)$. This means that the younger the participant the more likely he or she would be to engage in sufficiently vigorous activity. Educational level and job hours per week did not exhibit any significant relationship with activity levels. Finally, physical inactivity among Saudi adults according to age group was calculated and is shown in Fig. 2. The proportion of inactive Saudis was lower in the younger age categories (36.3\% and 36.4\% for age groups $15-29$ and 30-44 years, respectively). Inactivity then increased steadily with advancing age to reach the highest level (57.3\%) at age 60 years and above.

Table 2 Proportions (\%) of Saudi adults who are engaging in walking, moderate and vigorous physical activity for at least $10 \mathrm{~min}$ at a time, based on the numbers of days per week

\begin{tabular}{|c|c|c|c|c|c|c|c|c|c|}
\hline \multirow[b]{2}{*}{ Number of days per week } & \multicolumn{3}{|c|}{ Walking } & \multicolumn{3}{|c|}{ Moderate activity } & \multicolumn{3}{|c|}{ Vigorous activity } \\
\hline & All & Males & Females & All & Males & Females & All & Males & Females \\
\hline 0 & 22.3 & 19.9 & 26.9 & 42.6 & 51.1 & 26.3 & 71.7 & 63.8 & 87.7 \\
\hline 1 & 9.8 & 7.8 & 13.6 & 11.4 & 14.4 & 5.7 & 8.2 & 9.8 & 4.9 \\
\hline 2 & 9.2 & 9.7 & 8.3 & 11.7 & 12.7 & 9.5 & 6.4 & 8.3 & 2.6 \\
\hline 3 & 9.9 & 10.0 & 9.6 & 9.3 & 9.6 & 8.7 & 6.3 & 8.7 & 1.4 \\
\hline 4 & 4.1 & 4.0 & 4.2 & 2.6 & 2.7 & 2.2 & 2.5 & 3.9 & 0.0 \\
\hline 5 & 13.0 & 14.5 & 10.2 & 4.3 & 2.9 & 7.0 & 2.0 & 2.4 & 1.1 \\
\hline 6 & 2.4 & 2.6 & 2.0 & 0.9 & 1.0 & 0.8 & 0.9 & 1.0 & 0.6 \\
\hline 7 & 29.3 & 31.5 & 25.2 & 17.2 & 5.6 & 39.8 & 2.0 & 2.1 & 1.7 \\
\hline Total (\%) & 100.0 & 100.0 & 100.0 & 100.0 & 100.0 & 100.0 & 100.0 & 100.0 & 100.0 \\
\hline
\end{tabular}

Walking: $\chi^{2}=20.5$; two-sided level of significance $=0.005$.

Moderate activity: $\chi^{2}=221.7$; two-sided level of significance $=0.000$.

Vigorous activity: $\chi^{2}=75.1$; two-sided level of significance $=0.000$ 
Table 3 Proportions (\%) of Saudi adults who are engaging in walking, moderate and vigorous physical activity, based on the number of minutes per week

\begin{tabular}{|c|c|c|c|c|c|c|c|c|c|}
\hline \multirow{2}{*}{$\begin{array}{l}\text { Number of } \\
\text { minutes per week }\end{array}$} & \multicolumn{3}{|c|}{ Walking } & \multicolumn{3}{|c|}{ Moderate activity } & \multicolumn{3}{|c|}{ Vigorous activity } \\
\hline & All & Males & Females & All & Males & Females & All & Males & Females \\
\hline 0 & 23.5 & 21.3 & 27.9 & 43.6 & 51.9 & 27.5 & 72.3 & 64.4 & 88.3 \\
\hline $10-30$ & 10.8 & 11.5 & 9.4 & 10.1 & 13.3 & 4.1 & 5.5 & 6.4 & 3.8 \\
\hline $31-60$ & 10.8 & 10.5 & 11.4 & 8.1 & 9.7 & 4.8 & 6.0 & 7.5 & 2.8 \\
\hline $61-149$ & 21.6 & 18.9 & 22.8 & 9.5 & 9.9 & 6.5 & 6.6 & 8.6 & 2.6 \\
\hline $150-299$ & 14.1 & 17.1 & 12.3 & 9.1 & 8.6 & 12.3 & 5.8 & 8.9 & 1.2 \\
\hline 300 or more & 19.2 & 20.7 & 16.2 & 19.6 & 6.6 & 44.8 & 3.8 & 4.2 & 1.3 \\
\hline Total (\%) & 100.0 & 100.0 & 100.0 & 100.0 & 100.0 & 100.0 & 100.0 & 100.0 & 100.0 \\
\hline
\end{tabular}

Walking: $\chi^{2}=3.2$; two-sided level of significance $=0.021$.

Moderate activity: $\chi^{2}=32.1$; two-sided level of significance $=0.000$.

Vigorous activity: $\chi^{2}=71.6$; two-sided level of significance $=0.000$.

\section{Discussion}

The present study is the first to report on the physical activity profile of Saudis using the short-version telephone format of IPAQ. IPAQ is an international project supported by WHO and CDC. The main finding is that not many Saudi adults living in Riyadh were sufficiently vigorously active, based on 3 or more days per week of vigorous activity for at least 20 min or more per day. However, nearly half of the population was sufficiently moderately active, based on 5 or more days per week of moderate and walking activities for at least $30 \mathrm{~min}$ or more per day. In addition, inactivity prevalence among both sexes averaged 40.6\%, while the proportion of people meeting so-called health-enhancing physical activity levels was only $25.1 \%$. A previous survey estimated the prevalence of inactivity in Saudi Arabia to range from $43.3 \%$ to as high as $99 \%{ }^{13}$. Elsewhere, not many studies have reported physical activity data based on IPAQ. A Brazilian survey using the IPAQ short-form instrument found inactivity prevalence of $41.1 \%$ among Brazilian adults aged 20 years and above ${ }^{18}$.

Although differences in survey sampling and assessment methods make the task of comparing inactivity prevalence across countries extremely difficult, the current estimate of

Table 4 Physical activity profile of the Saudi adults (\%)

\begin{tabular}{lccc}
\hline Activity category & All & Males & Females \\
\hline 1. Inactive* $^{*}$ & 40.6 & 43.7 & 34.3 \\
2. Minimally active† & 34.3 & 36.5 & 30.1 \\
3. Activeł & 25.1 & 19.8 & 35.6 \\
\hline
\end{tabular}

$\chi^{2}=31.9 ;$ two-sided level of significance $=0.000$.

${ }^{\star}$ Not meeting criteria for minimally active or active.

† Meeting any of the following conditions: (1) participating in 3 or more days of vigorous-intensity activity for at least 20 min per day, or (2) participating in 5 or more days of moderate-intensity activity or walking for at least 30 min per day, or (3) participating in 5 or more days of any combination of walking, moderate-intensity or vigorous-intensity activities achieving a minimum of at least 600 MET-min per week (MET = metabolic equivalent).

¥ Meeting either of the following criteria: (1) vigorous-intensity activity on at least 3 days achieving at least 1500 MET-min per week, or (2) taking part in 7 or more days of any combination of walking, moderate-intensity or vigorous-intensity activities achieving a minimum of at least 3000 MET-min per week. inactivity level found in the present study appears similar to that reported from previous research using different measures of physical activity. Data from the Behavioral Risk Factors Surveillance System (BRFSS) in the USA indicated that the majority (54\%) of US adults were not physically active enough to meet the current recommendations of at least $30 \mathrm{~min}$ of moderate-intensity activity on most days of the week ${ }^{19}$. In another report analysing 19882002 BRFSS data in the USA, it was revealed that leisuretime physical inactivity decreased especially after 1996 in both men and women ${ }^{20}$. However, the US national health objectives for 2010 call for a reduction in the prevalence of no leisure-time physical activity to $20 \%{ }^{21}$.

The present investigation indicated that the proportion of men and women who walked for 150 min or more per week was $37.8 \%$ and $28.5 \%$, respectively. Brisk walking is a popular moderate type of aerobic activity. The cardiovascular benefits of brisk walking have been demonstrated in middle-aged and older women ${ }^{22}$. In the Women's Health Initiative study, walking briskly for at least 30 min on 5 days per week was associated with a $30 \%$ reduction in cardiovascular events over 3.2 years of follow-up ${ }^{22}$. In the USA it has been found that $33.7 \%$ of the

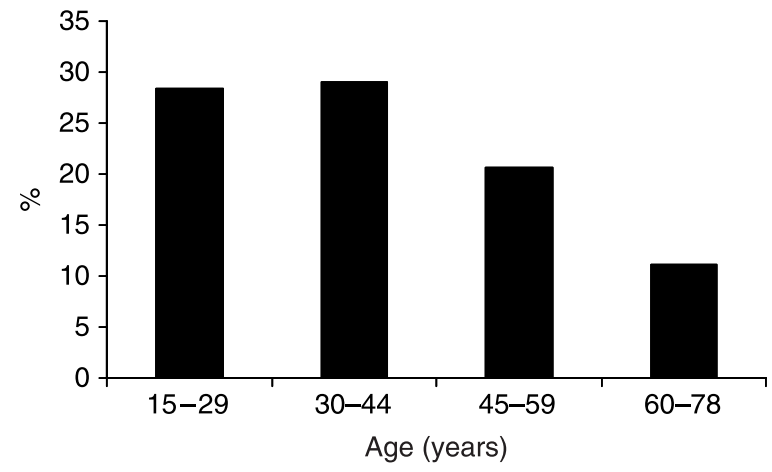

Fig. 1 The proportion of Saudi adults who are sufficiently active in health-enhancing physical activity according to age (participating in vigorous-intensity physical activity on at least 3 days achieving at least 1500 MET-min per week, or taking part in 7 or more days of any combination of walking, moderate-intensity or vigorous-intensity physical activities achieving a minimum of at least 3000 MET-min per week; MET = metabolic equivalent) 


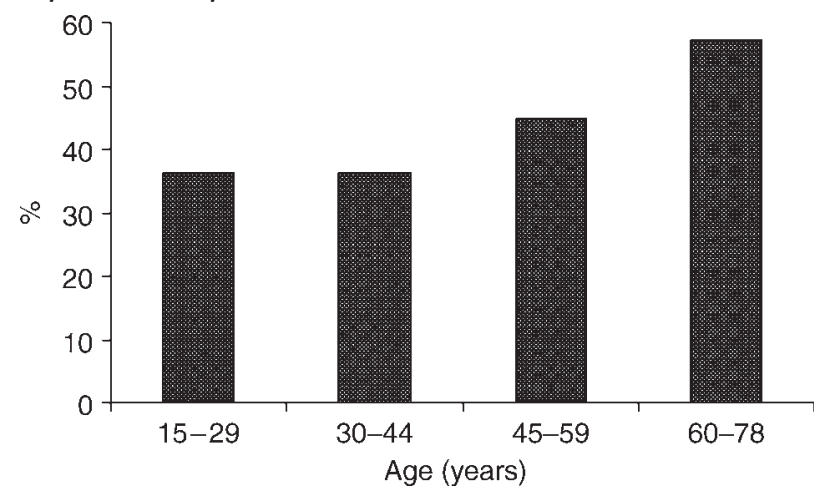

Fig. 2 Prevalence of physical inactivity among Saudi adults according to age

population were regular walkers, $45.6 \%$ occasional walkers and $20.7 \%$ never walkers ${ }^{23}$. Males were more regular walkers than females ${ }^{23}$. In the present study, the percentage of never walkers was $22.3 \%$, and more males were regular walkers than females.

The finding in the present report that females were more moderately active than males deserves some comment. The IPAQ instrument assessed all components of physical activity, including moderate-intensity activities done at home, such as carrying loads (e.g. babies), scrubbing floors, sweeping and vacuuming. These types of physical activity are more likely to be performed by female respondents, especially when considering that more than $80 \%$ of the females in our sample were not working. Another factor that might have contributed to the increased proportion of females doing more moderate activities than males is the fact that females in Saudi Arabia are more likely than males to use stationary exercise machines at home. The preceding discussion, however, cannot rule out overreporting of moderate-intensity physical activity by the female respondents in the present study. A recent study in Belgium using IPAQ found some overreporting of moderate physical activity ${ }^{24}$. Moreover, in the EUPASS (European Physical Activity Surveillance System) project, several physical activity measures, including IPAQ, were compared. The results showed that physical activity scores as well as overall caloric expenditure of IPAQ appeared rather high compared with previous studies and public health recommendations $^{25}$. However, the IPAQ scoring used in the present study is the one that has been modified recently and therefore is unlikely to be the reason for the high levels of moderate physical activity reported by the females. When moderate-, walking and vigorous-intensity physical activities were combined, females in the present study seemed to be more active than males. This was evident in the proportion of females who were classified into activity categories 2 and 3 (Table 4). However, most previous studies have shown that men are more active than women $^{18-20,26,27}$. Indeed, men usually report greater levels of total and vigorous physical activities, whereas women tend to report participating in low to moderate activities $^{3}$.

Contrary to the findings of the present study, several previous reports have shown that leisure-time physical inactivity is inversely associated with socio-economic status $^{26,28}$. In the USA, inactivity was shown to be more prevalent among less-educated people ${ }^{29}$. It is very likely that the disagreement between the present findings and the above cited studies may have resulted from the fact that previous reports assessed leisure-time physical activity. However, the IPAQ short-version telephone format, used in the present investigation, addresses all four components of physical activities, and is not limited to leisure-time physical activity. IPAQ short form, though, does not distinguish the pattern of physical activity behaviours based on such components.

The finding that physical inactivity increased in the older age group is not surprising. Age-related decline in physical activity has been described in the literature ${ }^{20,30}$. Data from the recent BRFSS study in the USA indicated that physical inactivity increased steadily with advancing age from 18-29 years to $70+$ years in both sexes ${ }^{20}$. However, a study conducted on a population of Nigerian civil servants found no significant physical activity trend across age between 20 and 64 years $^{26}$. A previous report on physical activity levels of Saudi males between the ages of 19 and 68 years found a curvilinear relationship between inactivity prevalence and age ${ }^{14}$. In that study, inactivity prevalence reached its highest level in the age group of 30-49 years, and the lowest level at age 60 and above. Again, the majority of the above-mentioned studies assessed mostly leisure-type physical activity and this may partially explain the seemingly different results reached regarding inactivity rates and age.

Finally, the present study is subject to the following limitations. First, this report used the short form of IPAQ intended for telephone interview. Thus, as is the case with any questionnaire, the respondents could have suffered from recall bias as well as social desirability bias. Second, the sample in the present study was randomly drawn from telephone book lists. However, a small percentage of the people in Riyadh city may not have telephone lines. Third, the sample distribution showed that there were more male than female respondents to the survey. The female/male ratio in the present sample is not in accordance with female/male census statistics for Saudis living in Riyadh. Having male interviewers may have partially contributed to the low female turn-out in this study.

From the findings of the present study, we can conclude that the prevalence of physical inactivity among Saudis living in Riyadh is relatively high, and that inactivity rate increases with age. Such high prevalence of inactivity represents a major public health concern. Therefore, public policies are needed to encourage active living and discourage sedentary habits. Health-care providers have an important role in promoting physical activity among the 
population. Furthermore, there is a need for a national study with a representative sample to address the issue of physical activity/inactivity in Saudi Arabia at large.

\section{Acknowledgements}

This research was supported by a grant from the Research Center, College of Education, King Saud University. The author acknowledges the assistance of Dr MA Sulaiman, M Dafterdar, M Al-Ahmadi, A Al-Sulaimi, A Al-Mangoor, F Al-Gohaize, S Al-Ghamidi, M Al-Bunyan, A Al-Amery, A Jabbari, S Al-Drees, A Al-Ghamidi and $\mathrm{K}$ Al-Khuraijee during data collection. Appreciation is also extended to Mrs Salwa Al-Rashid for back-translating IPAQ.

\section{References}

1 World Health Organization (WHO). The World Health Report 2003 - Shaping the Future. Geneva: WHO, 2003.

2 World Health Organization (WHO). The World Health Report 2002 - Reducing Risks, Promoting Healthy Life. Geneva: WHO, 2002.

3 US Department of Health and Human Services. Physical Activity and Health: A Report of the Surgeon General. Atlanta, GA: Centers for Disease Control and Prevention, National Centers for Chronic Disease Prevention and Health Promotion, 1996.

4 Fletcher G, Balady G, Blair S, Blumenthal J, Caspersen C, Chaitman B, et al. Statement on exercise: benefits and recommendations for physical activity programs for all Americans. Circulation 1996; 94: 857-62.

5 Pate R, Pratt M, Blair S, Haskell W, Macera C, Bouchard C, et al. Physical activity and public health. A recommendation from the Centers for Disease Control and Prevention and the American College of Sports Medicine. Journal of the American Medical Association 1995; 273: 402-7.

6 Katzmarzyk P, Janssen I, Ardern C. Physical inactivity, excess adiposity and premature mortality. Obesity Reviews 2003; 4: 257-90.

7 Giannuzzi P, Mezzani A, Saner H, Bjornstad H, Fioretti P, Mendes M, et al. Physical activity for primary and secondary prevention. Position paper of the Working Group on Cardiac Rehabilitation and Exercise Physiology of the European Society of Cardiology. European Journal of Cardiovascular Prevention and Rebabilitation 2003; 10: 319-27.

8 American College of Sports Medicine. ACSM's Guidelines for Exercise Testing and Prescription. Baltimore, MD: Lippincott Williams \& Wilkins, 2000.

9 Macera C, Pratt M. Public health surveillance of physical activity. Research Quarterly for Exercise and Sport 2000; 71(Suppl.): 97-103.

10 World Health Organization (WHO). Global Strategy on Diet, Physical Activity and Health. 57th World Health Assembly, WHA57.17. Geneva: WHO, 2004.

11 Al-Hazzaa H. Physical activity, fitness and fatness among Saudi children and adolescents: implications for cardiovascular health. Saudi Medical Journal 2002; 23: 144-50.

12 Al-Hazzaa H. Patterns of physical activity among Saudi children, adolescents and adults with special reference to health. In: Musaiger A, Miladi S, eds. Nutrition and Physical Activity in the Arab Countries of the Near East. Manama: Bahrain Center for Studies and Research, 2000; 109-27.

13 Al-Hazzaa H. Prevalence of physical inactivity in Saudi Arabia: a brief review. East Mediterranean Health Journal 2004; 10: 663-70.
14 Al-Refaee S, Al-Hazzaa H. Physical activity profile of adult males in Riyadh city. Saudi Medical Journal 2001; 22: $784-9$.

15 Al-Hazzaa $\mathrm{H}$. The public health burden of physical inactivity in Saudi Arabia. Journal of Family and Community Medicine 2004; 11: 45-52.

16 Craig C, Marshall A, Sjostrom M, Bauman A, Booth M, Ainsworth B, et al. International Physical Activity Questionnaire: 12 -country reliability and validity. Medicine and Science in Sports and Exercise 2003; 35: 1381-95.

17 Ainsworth B, Haskell W, Whitt M, Irwin M, Swartz A, Strath S, et al. Compendium of physical activity: an update of activity codes and MET intensities. Medicine and Science in Sports and Exercise 2000; 32(Suppl.): S498-516.

18 Hallal P, Vector C, Wells J, Lima R. Physical inactivity: prevalence and associated variables in Brazilian adults. Medicine and Science in Sports and Exercise 2003; 35: 1894-900.

19 Centers for Disease Control and Prevention. Prevalence of physical activity, including lifestyle activities among adults United States, 2000-2001. Morbidity \& Mortality Weekly Report 2003; 52: 764-9.

20 Centers for Disease Control and Prevention. Prevalence of no leisure-time physical activity - 35 states and district of Columbia, 1988-2002. Morbidity \& Mortality Weekly Report 2004; 53: 82-6.

21 US Department of Health and Human Services (DHSS). Healthy People 2010: National Health Promotion and Disease Prevention Objectives. Washington, DC: DHHS, 2000.

22 Manson J, Greenland P, LaCroix A, Stefanick M, Moulton C, Oberman A, et al. Walking compared with vigorous exercise for the prevention of cardiovascular events in women. New England Journal of Medicine 2002; 347: 716-25.

23 Eyler A, Brownson R, Bacak S, Housemann R. The epidemiology of walking for physical activity in the United States. Medicine and Science in Sports and Exercise 2003; 35: 1529-36.

24 Rzewnicki R, Auweele Y, DeBourdeaudhuij I. Addressing overreporting on the International Physical Activity Questionnaire (IPAQ) telephone survey with a population sample. Public Health Nutrition 2003; 6: 299-305.

25 Rutten A, Ziemainz H, Schena F, Stahl T, Stiggelbout M, Auweele $\mathrm{Y}$, et al. Using different physical activity measurements in eight European countries: results of the European Physical Activity Surveillance System (EUPASS) time series survey. Public Health Nutrition 2003; 6: 371-6.

26 Forrest K, Bunker C, Kriska A, Ukoli F, Huston S, Markovic N. Physical activity and cardiovascular risk factors in a developing population. Medicine and Science in Sports and Exercise 2001; 33: 1598-604.

27 Martin S, Morrow I, Jackson A, Dunn A. Variables related to meeting the CDC/ACSM physical activity guidelines. Medicine and Science in Sports and Exercise 2000; 32: 2087-92.

28 Macera C, Croft J, Brown D, Ferguson T, Lane M. Predictions of adopting leisure-time physical activity among a biracial community cohort. American Journal of Epidemiology 1995; 142: 629-35.

29 Crespo C, Ainsworth B, Keteyian S, Heath G, Smit E. Prevalence of physical inactivity and its relation to social class in US adults: results from the Third National Health and Nutrition Examination Survey, 1988-1994. Medicine and Science in Sports and Exercise 1999; 31: 1821-7.

30 Caspersen C, Pereira M, Curran K. Changes in physical activity patterns in the United States, by sex and crosssectional age. Medicine and Science in Sports and Exercise 2000; 32: 1601-9. 